\title{
On the embedded warning system based on wireless sensor networks for marine monitoring and defending
}

\author{
YUE Xiangyu \\ School of Management and Engineering, Nanjing University; Nanjing Jiangsu; 210008; PR China
}

Email address:

yuexiangyu168@gmail.com

\section{To cite this article:}

YUE Xiangyu. On the Embedded Warning System Based on Wireless Sensor Networks for Marine Monitoring and Defending. American Journal of Embedded Systems and Applications. Vol. 1, No. 1, 2013, pp. 23-26. doi: 10.11648/j.ajesa.20130101.14

\begin{abstract}
The warning system for ocean monitoring and defending is a remote video ocean monitoring system engineering using the information gathering technologies, such as ultrasonic, radar, laser, infrared, machine vision, interactive, and so on, and other integrated technologies, such as wireless sensor networks, modern communication, computer information processing, solar energy. The engineering, based on information collecting technology and wireless sensor networks and combined with the technologies such as Beidou navigation and positioning, images and meteorological sensor information, geographic information, remote sensing information, forms a marine resource digital information management system that provides a full range of electronic monitoring and protection for the oceans. The wireless sensor network is a self-organizing network composed of a large number of sensor nodes, which sets such three technologies as the sensors, micro-electromechanical systems and network in one. The network's aim is to sense, collect and process the information of the objects in the network coverage and send it to the data processing center to provide the basis for ocean monitoring and managing and protecting the marine safety. With the continuous development of micro-electromechanical system MEMS, wireless communication technology and electronic technology, the practical field of wireless sensor networks is becoming wider and wider.
\end{abstract}

Keywords: Embedded System, Wireless Sensor Networks, Beidou Positioning System, Wireless Communication, Detection, Warning

With the growing increase of China's comprehensive national strength as well as the continued exploration and development of marine resources, marine monitoring and early warning system are being gradually demanded and valued by people. However, due to the huge ocean area, it has more difficulty to control. This article designed an embedded marine monitoring and early warning system based on wireless sensor networks which can play a good role in marine monitoring and marine security protection.

\section{Current Research Status of Wireless Sensor Network at Home and Abroad}

Wireless sensor network has important scientific value and broad application prospects which also causes a great concern of many countries' military department, industry and academia. Representatives of which is the "Smart Dust" laboratory jointly established by the University of California at Berkeley and Intel Corporation. Its goal is to make the U.S. military's surveillance of the activities of the enemy without being noticed. U.S. Army proposed the "Smart Sensor Network Communication" program in 2001. It connected the unattended type of ammunition, sensors and robotic systems in Future Combat Systems into a network so that exponentially increased the ability of a single sensor, while improving the system's efficiency and robustness. Intel Corporation also issued a "new computing development plan based on micro-sensor networks "in 2002, dedicated to the application of sensor networks in the fields of environmental monitoring, forest fire fighting, Haiti sector etc. Research institutions in many other countries such as Britain , Germany, Canada, Japan have also joined the research of wireless sensor network, major companies includes Philips, France Telecom, Siemens, NEC, Omron, Sky2 and so on.

Domestic research on wireless sensor network technology is still in its infancy. Compared with foreign countries, there is still a long way to go. In the theoretical research, Tsinghua University and other universities and the CAS etc. all have had some preliminary research on network security 
technology, energy technology and other core processing technology, routing algorithms and communication protocols and so on. In practical applications, the program that combines the wireless communications technology and sensor technology appeared even later. For instance, wireless sensor network node hardware and software platforms for monitoring the temperature, humidity and soil $\mathrm{pH}$ inside the greenhouse of vegetables, developed by the CAS Institute of Computing Ningbo segments which based on ZigBee protocol and a dedicated low-power processor chips and intelligent health monitoring system for major engineering structures developed by academicians $\mathrm{Ou}$ Jinping etc. of Harbin Industrial University. In addition, some of the other domestic universities and research institutions have also made some achievements on wireless sensor network technology. But there is less innovative research on the system and there are many problems, so the level of research is relatively backward. Therefore, future research and application in this area should be strengthened to narrow the gap between foreign countries of technologies in this field.

\section{Key Technologies of Wireless Sensor Networks in Marine Monitoring and Defending early Warning System}

The wireless sensor network in ocean monitoring and defending early warning system is a wireless infrastructure network. Compared with the wireless ad hoc networks, it has many defects in the aspects of resources and so on. To compensate for these shortcomings, we have taken the following key measures in the research and design to the wireless sensor network:

\subsection{Increase Self-Management Skills and Self-Organizing Capacity of the Wireless Sensor Network}

The application of the wireless sensor networks in ocean monitoring and defending early warning system will be impacted and restricted by the surrounding environment. The main structure wireless sensor networks used is a wireless ad hoc network structure, and its sensor nodes are placed in the monitoring area. So it must be able to automatically launch and must take the initiative in the signal, contacting with an adjacent node when having information, and then feedback the acquired position and work status of the adjacent nodes to the work station. Work station will then plan the network topology structure according to the recorded information.

\subsection{Strengthen Energy Management in Wireless Sensor Networks}

As the network nodes of the wireless sensor in marine monitoring and defending early warning system is limited by the battery to supply energy, the use of energy must be accurately calculated which could make them reasonably switch between dormancy and working conditions, thus to achieve the purposes of saving energy as much as possible, reducing space and a reduction of cost.

\subsection{Optimize Sensor Network's Data Management, Processing and Query}

The wireless sensor network in ocean monitoring and defending early warning system is data-centric, so data storage, management, processing and query are its core technologies. This is mainly manifested in the following aspects:

(1) In the application of wireless sensor networks in ocean monitoring and defending early warning system, monitoring data and information is of the most importance. However, the working condition of each specific node is not the main part.

(2) Because marine monitoring and defending early warning system will monitor too large scope and too many nodes, so the data collection is very large and complex, and thus it has a feature of redundancy .

(3) For the purpose of data processing by data- centric wireless sensor network, sensory data management becomes one of the core technologies of ocean monitoring and defending early warning system, including sensory data storage, query, analysis, mining, understanding, decision analysis and many other aspects.

(4) Due to the large quantity of wireless sensor network nodes in marine monitoring and defending early warning system, when network bandwidth is not very satisfactory, energy issues are of the most importance.

(5) Since the attribute addressing manner of the wireless sensor network in ocean monitoring and defending early warning system is node allocation address, there is no need to assign each node a unique address.

\subsection{Strengthen the Node Localization of the Wireless Sensor Network}

When the ocean monitoring and defending early warning system is applying to the wireless sensor network, most critically, the observer should make the right data processing to the monitored areas based on collected data information, and the data information collected in each node must have a comprehensive consideration on the information in the coordinate system of measured position.

Only in this way can improve the routing efficiency, therefore to grasp the load balancing and topology structure of network better. Meanwhile, during the formation of the algorithm, it must be carefully considered about the issues like how the network of each node generates its own spatial coordinates and so on. We use Beidou navigation and positioning technologies, the world's best satellite navigation and positioning system, to position each node. 


\subsection{Construct Protocol Stack for Sensor Network}

The protocol stack for wireless sensor network of ocean monitoring and defending early warning system includes five layers of protocols: the physical layer, the network layer, the data link layer, the transport layer and the application layer. This five-layer respectively correspond to each protocol layer of the Internet protocol stack. Additionally, the protocol stack for wireless sensor network also includes the following three management platforms: energy management platform, task management platform and mobile management platform.

In the protocol stack for wireless sensor network of the marine monitoring and defending early warning system, location and time synchronization sub-layer are the most important. It will not only provide information support to other wireless sensor network protocol layers, but also make cooperative positioning and time synchronous negotiation relying on the data transmission channel. In each protocol layer, QoS management designs queue management, priority mechanism or bandwidth reservation mechanism etc., and gives special treatment to the specific application data. Topology control achieves topology generation through the link layer, physical layer and routing layer, which also provides basic information support to optimize the MAC protocol process, and reduce the energy consumption of network, improving the efficiency of agreement. Wireless sensor network management then requires each protocol layers to not only conveniently embed a variety of information interfaces but also be able to regularly collect traffic information and operating status of the protocol as well as coordinate all protocol components in the control network, which can make it operate normally.

\section{Implementations of the Monitoring Node for Wireless Sensor Network in Ocean Monitoring and Defending early Warning System}

The monitoring node for wireless sensor network in ocean monitoring and defending early warning system is mainly responsible for the monitoring and data collection of crashers throughout the ocean. Its core is the low-power, 32-bit chip STM32F103 ZET6 based on the ARM Cortex-M3 kernel, which combined with wireless transceiver chip CC2420. The mode of surveillance and information gathering technology includes ultrasonic wave, radar, laser, infrared ray, machine vision, and interaction and so on. The information processing, however, mainly analyzes and processes the collected information in the ARM chip. And information determination is a dynamic and real-time measurement and identification of risk or security status based on the timing, the weather, and the information such as the distance, relative acceleration, relative speed etc. between the detection node and monitoring object. When the information is identified and judged as dangerous, the main responsibility for warning information is to calculate the risk level and dangerous direction, and give appropriate sound and light alarm as well as transmit information through a wireless sensor network, notifying the control room located onshore. The monitoring node is battery-powered. According to the performance requirements of marine monitoring, ultrasonic sensors, infrared sensors, radars, cameras etc. can be used to gather information. Additionally, if it is aiming to detect partial marine ecological information in the use of this system in the meanwhile, salinity sensors, temperature and humidity sensors can be also installed on it. For example, using temperature and humidity sensor SHTll, it employs advanced CMOSensTM digital sensor technology. The stability and reliability of which is relatively high. The interface mode for the sensor is two-wire digital mode, which can be directly connected to the microcontroller so that its peripheral circuit has been greatly simplified. The package is SMD chip which greatly reduces the area of $\mathrm{PCB}$.

\subsection{Structure of the Monitoring Node for Wireless Sensor Network}

The main function of the monitoring node for wireless sensor network in ocean monitoring and defending early warning system is to monitor and collect data of marine suspicious object and then transfer it to the base station node. It consists of four parts-the sensor module, the processing module, the transmission module and the power supply module. The sensor module uses an interactive electronic whiteboard including electromagnetic induction, infrared sensing and ultrasonic sensing, which connects the processing module by simulating $112 \mathrm{C}$ interface. The processing module controls the routing protocols and the node's power management etc. between other modules and the wireless sensor network. The transmission module mainly consists of a low-power wireless communication chip CC2420 and its peripheral circuits. The software runs the appropriate communication protocols. The energy supply module uses two 5 AA solar rechargeable batteries.

\subsection{Hardware Design of the Monitoring Node for Wireless Sensor Network}

\subsubsection{Processing Circuit Module}

STM32F103ZET6, selected for the monitoring node for wireless sensor network in ocean monitoring and defending early warning system, is a low-power 32-bit microcontroller based on the ARM Cortex-M3 kernel. The internal structure of this type of chips is Harvard with the advantages of low power consumptions and abundant resources etc. The choice of storage is a $512 \mathrm{~KB}$ on-chip ROM and a 64KB SRAM. In addition, this chip has $112 \mathrm{IO}$ ports, three 12-bit ADC channels, a 12-bit DAC, two basic timers, four general purpose timers and two senior timers, which can work normally in a variety of different modes. It also has an SDIO interface, an FSMC interfaces, three SPI, two I2C bus interfaces as well as multiple serial ports and CAN bus 
interfaces, which can be designed by two programming approaches-ISP and JTAG. It supports three kinds of low-power mode, resulting in a best balance point among low power consumption, short startup time and available wakeup sources.

\subsubsection{Transmission Circuit Module}

The selection of the RF transceiver of the monitoring node for wireless sensor network in ocean monitoring and defending early warning system is CC2420, coming from the SmartRF03 technology of Chipcon, which is made by 0.18 um CMOS process. It has many advantages such as minimal external components, very low power consumptions, relatively stable performance, low working voltage $(2.1 \sim 3.6 \mathrm{v})$, small volume etc. Therefore it is quite suitable for integration. Using this chip in wireless sensor network in ocean monitoring and defending early warning system, data transfer rates can be up to $25 \mathrm{OKbPs}$, resulting in achieving rapid multipoint networking. CC2420 sets the operation mode of 4-wire SPI (SI, S0, SCLK, CSn) bus chip, realizing functions such as read (write) functions of the cached data and status register etc. The transmit/receive buffer is set by controlling the state of FIFO's and FIFOP's pin interface.

\subsubsection{Sensor Circuit Module}

The sensor module can integrate a variety of sensors into one board, which makes it with a high degree of integration. It integrates all functions like infrared sensing, ultrasonic sensing, temperature and humidity sensing, signal conversion, A/D conversion etc. into one single chip, resulting in free and flexible mode choosing and high energy saving ability. Especially, after monitoring and communication, the sensor module can be automatically transferred into the low-power mode which greatly reduces the energy consumption.

\section{Brief Introduction to the Author}

Yue Xiangyu (1992- ), who is a male, Han nationality, was born in Weifang, Shandong, with BA, Nanjing University, mainly engaged in electrical information and automation, aesthetics and other aspects of learning and research, specializing in modern industrial embedded control systems, networked control systems, intelligent control. He presided over one national college student science and technology innovation project, taking part in the research on one "Eleventh Five-Year Plan" education and science key project of the Education Ministry, and one soft science research project of Shandong Province. He has 12 science and technology papers published in Chinese and oversea academic journals. He has won the 1st scholarship of China, the 1st scholarship of people, the 1st and the 2nd prizes of China Education Robotics Competition, the 1st prize of China Mathematical Modeling Contest, the top award of scientific research achievement of Nanjing University, two China science and technology patents and the honorary title, "three-good-student" of Jiangsu Province. In addition, as a representative of Nanjing University, he went to the National University of Singapore to participate in an academic exchange program. Address: School of Management and Engineering, Xianlin Campus of Nanjing University, 163, Xianlin Avenue, Qixia District, Nanjing, Jiangsu Province, PR China; Zip: 210008.

\section{References}

[1] Ye Tao, Conghua Lan (2011), "Research on the Forest Disaster Warning System Based on Wireless Sensor Networks", Computer Knowledge and Technology 7.

[2] Xiaoqiang Yi, Jing Zhao (2011), "Research on Anti-Rear-End Collision Warning System”, Technological Development of Enterprise 12, pp.8-9.

[3] Xuejia Cai, Xu Li, Feng Deng(2011), "Research on Embedded Remote Control System Based on Wireless Sensor Networks", Modern Electronic Technology 16, pp.96-98. 